Louisiana State University

LSU Digital Commons

Faculty Publications

Department of Chemistry

11-17-2009

\title{
Neutron scattering study of the dynamics of a polymer melt under nanoscopic confinement
}

\author{
Margarita Krutyeva \\ Forschungszentrum Jülich (FZJ) \\ Jaime Martin \\ CSIC - Instituto de Ciencia y Tecnologia de Polimeros (ICTP) \\ Arantxa Arbe \\ CSIC UPV Centro de Fisica de Materials CFM \\ Juan Colmenero \\ CSIC UPV Centro de Fisica de Materials CFM \\ Carmen Mijangos \\ CSIC - Instituto de Ciencia y Tecnologia de Polimeros (ICTP)
}

See next page for additional authors

Follow this and additional works at: https://digitalcommons.Isu.edu/chemistry_pubs

\section{Recommended Citation}

Krutyeva, M., Martin, J., Arbe, A., Colmenero, J., Mijangos, C., Schneider, G., Unruh, T., Su, Y., \& Richter, D. (2009). Neutron scattering study of the dynamics of a polymer melt under nanoscopic confinement. Journal of Chemical Physics, 131 (17) https://doi.org/10.1063/1.3258329

This Article is brought to you for free and open access by the Department of Chemistry at LSU Digital Commons. It has been accepted for inclusion in Faculty Publications by an authorized administrator of LSU Digital Commons.

For more information, please contact ir@lsu.edu. 
Authors

Margarita Krutyeva, Jaime Martin, Arantxa Arbe, Juan Colmenero, Carmen Mijangos, Gerald J. Schneider, Tobias Unruh, Yixi Su, and Dieter Richter 


\section{Neutron scattering study of the dynamics of a polymer melt under nanoscopic confinement}

Margarita Krutyeva, Jaime Martin, Arantxa Arbe, Juan Colmenero, Carmen Mijangos, Gerald J. Schneider, Tobias Unruh, Yixi Su, and Dieter Richter

Citation: The Journal of Chemical Physics 131, 174901 (2009); doi: 10.1063/1.3258329

View online: https://doi.org/10.1063/1.3258329

View Table of Contents: http://aip.scitation.org/toc/jcp/131/17

Published by the American Institute of Physics

\section{Articles you may be interested in}

Polymer dynamics under cylindrical confinement featuring a locally repulsive surface: A quasielastic neutron scattering study

The Journal of Chemical Physics 146, 203306 (2017); 10.1063/1.4974836

Reptation of a Polymer Chain in the Presence of Fixed Obstacles

The Journal of Chemical Physics 55, 572 (1971); 10.1063/1.1675789

Description of poly(ethylenepropylene) confined in nanopores by a modified Rouse model

The Journal of Chemical Physics 146, 203309 (2017); 10.1063/1.4975977

Reduced-mobility layers with high internal mobility in poly(ethylene oxide)-silica nanocomposites

The Journal of Chemical Physics 146, 203303 (2017); 10.1063/1.4974768

Comment on "Neutron scattering study of the dynamics of the polymer melt under nanoscopic confinement" [ $\mathrm{J}$. Chem. Phys. 131, 174901 (2009)]

The Journal of Chemical Physics 134, 057101 (2011); 10.1063/1.3532077

A Theory of the Linear Viscoelastic Properties of Dilute Solutions of Coiling Polymers

The Journal of Chemical Physics 21, 1272 (1953); 10.1063/1.1699180

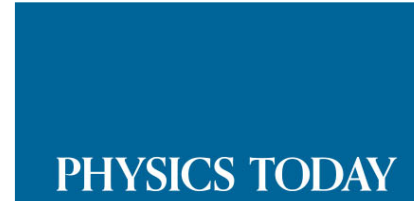

WHITEPAPERS
ADVANCED LIGHT CURE ADHESIVES

READ NOW

Take a closer look at what these environmentally friendly adhesive systems can do
PRESENTED BY

QMASTERBOND: 


\title{
Neutron scattering study of the dynamics of a polymer melt under nanoscopic confinement
}

\author{
Margarita Krutyeva, ${ }^{1, a)}$ Jaime Martin, ${ }^{2}$ Arantxa Arbe, ${ }^{3}$ Juan Colmenero, ${ }^{3,4}$ \\ Carmen Mijangos, ${ }^{2}$ Gerald J. Schneider, ${ }^{5}$ Tobias Unruh, ${ }^{6}$ Yixi Su, ${ }^{5}$ and Dieter Richter ${ }^{1}$ \\ ${ }_{1}^{1}$ Institut für Festkörperforschung, Forschungszentrum Jülich, Jülich 52425, Germany \\ ${ }^{2}$ Instituto de Ciencia y Tecnología de Polímeros, CSIC, 28066 Madrid, Spain \\ ${ }^{3}$ Centro de Física de Materiales (CSIC-UPV/EHU), Materials Physics Center (MPC), \\ 20080 San Sebastián, Spain \\ ${ }^{4}$ Departamento de Física de Materiales, UPV/EHU, 20080 San Sebastián, Spain and \\ Donostia International Physics Center, 20080 San Sebastián, Spain \\ ${ }^{5}$ Jülich Centre of Neutron Research (JCNS) at FRM II, Garching 85747, Germany \\ ${ }^{6}$ Forschungsneutronenquelle Heinz, Maier-Leibnitz, (FRM II), Garching 85747, Germany
}

(Received 9 June 2009; accepted 14 October 2009; published online 4 November 2009)

Poly(ethylene oxide) confined in an anodic aluminum oxide solid matrix has been studied by different neutron scattering techniques in the momentum transfer $(\vec{Q})$ range $0.2 \leq Q=|\vec{Q}|$ $\leq 1.9 \AA^{-1}$. The cylindrical pores of the matrix present a diameter $(40 \mathrm{~nm})$ much smaller than their length $(150 \mu \mathrm{m})$ and are parallel and hexagonally ordered. In particular, we investigated the neutron intensity scattered for two orientations of the sample with respect to the incident beam, for which the $\vec{Q}$ direction was either parallel or perpendicular to the pores for a scattering angle of $90^{\circ}$. Diffuse neutron scattering at room temperature has shown that the aluminum oxide has amorphous structure and the polymer in the nanoporous matrix is partially crystallized. Concerning the dynamical behavior, for $Q<1 \AA^{-1}$, the spectra show Rouse-like motions indistinguishable from those in the bulk within the uncertainties. In the high- $Q$ limit we observe a slowing down of the dynamics with respect to the bulk behavior that evidences an effect of confinement. This effect is more pronounced for molecular displacements perpendicular to the pore axis than for parallel displacements. Our results clearly rule out the strong corset effect proposed for this polymer from nuclear magnetic resonance (NMR) studies and can be rationalized by assuming that the interactions with the pore walls affect one to two adjacent monomer monolayers. (C) 2009 American Institute of Physics.

[doi:10.1063/1.3258329]

\section{INTRODUCTION}

The synthesis of nanocomposite materials is an intensively developing area of science aiming at the production of materials with novel properties. Because of their peculiar features, such as macromolecular architecture and unique viscoelastic properties, polymers are used as a basis for nanocomposites. ${ }^{1}$ The emergence of confinement effects on the polymer dynamics in these systems motivated the study of their influence on the physical and chemical properties of polymer systems. In particular, linear polymer melts confined in straight nanoporous aluminum oxide channels can form tailored one-dimensional structures, from nanotubes to nanorods, depending on the wetting on the surface of the pore walls. ${ }^{2}$ Many of the final properties of these systems are determined by the dynamical properties of the polymer. With this work we contribute to these questions by investigating the influence of confinement on the dynamics of a linear polymer melt. We note that in particular the study of confinement effects on the glass dynamics of polymers has been a much investigated topic during the last few years (see, e.g., Refs. 3-6).

${ }^{a)}$ Electronic mail: m.krutyeva@fz-juelich.de.
Polymer dynamics is very rich and shows different phenomena depending on the temperature and length scale of observation. Close and above the glass transition temperature $T_{\mathrm{g}}$, the dynamics is dominated by the $\alpha$-relaxation at intermolecular level. Increasing the temperature, chain mobility is enhanced and, at length scales larger than those corresponding to the statistical segment, the chain dynamics can be well described by the Rouse model. In this temperature range, the viscoelastic properties of polymers arise due to the macromolecular character of the structural units in the system. The challenge then lays in the description of many-particle correlations in polymer systems which are consequence of the uncrossibility of polymer chains. The effects of these correlations-known as entanglements-become strong if the molecular mass of the polymer is larger than some critical value $M_{\mathrm{c}}$. In the pioneering works of de Gennes, ${ }^{7}$ and Doi and Edwards, ${ }^{8}$ the successful reptation/tube theory was presented. The theory introduces the concept of a fictitious "tube" and developed the mathematical background of the model. The tube-concept looks simple and clear. The main parameter of the theory - the tube diameter - can be obtained from mechanical measurements and, microscopically, from neutron scattering experiments. ${ }^{9,10}$ In general, the tube diam- 
eter is found to be between 46 and $100 \AA$. Though in general the reptation predictions for a number of experimental observables like viscosity or translational diffusion coefficient are qualitatively in good agreement with experiment, deviations are evident. To explain them, various modifications of the reptation theory have been proposed that mainly intend to take into account so-called "end" effects (constraint release) and fluctuations of primitive path of polymer chain (contour length fluctuations). ${ }^{8,9,11,12}$ Furthermore, the question about the transition from unentangled Rouse behavior ${ }^{13}$ to entangled dynamics still remains under consideration.

The dynamics of poly(ethylene oxide) (PEO) chains under confinement in nanoporous matrices have recently been studied by field-cycling nuclear magnetic resonance (NMR) relaxometry. ${ }^{14}$ The variation of both the molecular weight of the polymer melt and the size of the nanoporous matrix channel allowed exploring the dynamics at different Flory radius/pore size ratios. It was found that the dynamics of the confined polymer was very different from that in the bulk. The most striking finding was that the confined dynamics essentially did not depend on the molecular weight of the polymer and the relation between Flory radius $R_{\mathrm{F}}$ and size of the pore channel. This finding was termed "corset effect" and was declared to appear as a consequence of the low compressibility of the polymer melt, uncrossibility of the polymer chains, and impenetrable pore walls. The estimation of the effective tube diameter gives the value $0.5 \mathrm{~nm}$, i.e., one order of magnitude smaller than that obtained from neutron scattering experiments in the corresponding polymer bulk. ${ }^{15}$ It has also been reported that the effect of confinement is seen already if $R_{\mathrm{F}} \ll a$, where $a$ is the confinement size. The last field-cycling NMR relaxation experiments claim a visible difference between the dynamics of bulk polymer and that under confinement, already at pore sizes 100 times larger than Flory radius. ${ }^{16}$

Neutron scattering accesses dynamical properties with space/time resolution. For polymer dynamics, this allows separating different dynamical regimes for different length scales. ${ }^{17}$ In this work we report a study of the hydrogen self-correlation function in protonated PEO confined in nanoporous anodic aluminum oxide (AAO) templates. The AAO templates are characterized by a well-defined geometry. The pore system consists of parallel channels of a given diameter with narrow size distribution. The length of the channels is much larger than their diameter, allowing to neglect any end effects of the external surface of the templates. The polymer dynamics has been studied by neutron scattering using backscattering (BS) and time-of flight (ToF) spectrometers. These instruments allowed to explore the time window from picoseconds to a few nanoseconds and values of moment transfer $Q=|\vec{Q}|$ in the interval $0.17 \AA^{-1}<Q$ $<1.9 \AA^{-1}$ corresponding roughly to length scales from a few nanometers down to some tenth of a nanometer. We aimed to explore the influence of the confinement on polymer dynamics and to check whether any interaction between the polymer and pore walls is visible in the dynamics.

\section{THEORY}

\section{A. Neutron scattering}

Neutron scattering experiments measure the intensity scattered into a solid angle between $\Omega$ and $\Omega+\Delta \Omega$ as a function of the energy difference between the incident $(E)$ and scattered $(E+\Delta E)$ neutrons. For protonated samples the cross section is dominated by the incoherent scattering from the hydrogens and the incoherent scattering function $S_{\text {inc }}(\vec{Q}, \omega)$ is revealed. Here $\vec{Q}$ is the momentum transfer and $\hbar \omega=\Delta E$. $S_{\text {inc }}(\vec{Q}, \omega)$ is the Fourier transformation of the self-part of the Van Hove correlation function $G_{\text {self }}(\vec{r}, t)$ with respect to time and space:

$$
S_{\text {inc }}(\vec{Q}, \omega) \propto \int_{-\infty}^{\infty} \int_{-\infty}^{\infty} G_{\text {self }}(\vec{r}, t) \exp (i(\vec{Q} \vec{r}-\omega t)) d \vec{r} d t,
$$

where

$$
G_{\text {self }}(\vec{r}, t)=\frac{1}{N}\left\langle\sum_{i=1}^{N} \delta\left(\vec{r}-\left(\vec{r}_{i}(t)-\vec{r}_{i}(0)\right)\right)\right\rangle .
$$

$G_{\text {self }}(\vec{r}, t)$ gives the probability to find a given nucleus $i$ around the position $\vec{r}+d \vec{r}$ if it was located at $\vec{r}_{i}(0)$ at $t=0$. Thus, incoherent scattering gives information about the correlations between the positions of the same nucleus (usually hydrogen) at different times.

\section{B. General approach to spectra analysis}

In the $Q$-range usually explored by cold ToF and BS techniques $\left(0.2 \AA^{-1} \leq Q=|\vec{Q}| \leq 1.9 \AA^{-1}\right)$ the slow decay of the incoherent intermediate scattering function $S_{\text {inc }}(Q, t)$ of glass-forming systems attributed to segmental relaxation above $T_{\mathrm{g}}$ is generally analyzed in terms of an empirical stretched exponential or Kohlrausch-Williams-Watts (KWW) function:

$$
S_{\text {inc }}^{\mathrm{KWW}}(Q, t)=A(Q) \exp \left(-\left(\frac{t}{\tau_{\mathrm{KWw}}(Q, T)}\right)^{\beta}\right),
$$

where $\tau_{\mathrm{KWW}}$ is the relaxation time that depends on $Q$ and temperature, $0 \leq \beta \leq 1$ is stretching parameter, and $A(Q)$ is the Debye Waller factor. It has been found for different polymers that in the $Q$-regime $0.2-1 \AA^{-1} \tau_{\mathrm{KWW}}$ follows the power law

$$
\tau_{\mathrm{KWW}} \propto Q^{-2 / \beta} .
$$

The power law (4) is related to a Gaussian form of the selfpart of Van Hove correlation function [Eq. (2)]. ${ }^{18}$ Moreover, for most of the polymers, $\beta$ is experimentally found to be of the order of 0.5 and the power law $\tau_{\mathrm{KWW}} \propto Q^{-4}$ is rather well fulfilled in the limit of low $Q$-values $\left(Q \leq 1 \AA^{-1}\right) .{ }^{17}$ At higher $Q$-values $\left(\geq 1 \AA^{-1}\right)$ deviations from Eq. (4) become pronounced, indicating non-Gaussian dynamics at short scales. ${ }^{19}$

\section{Rouse theory}

The chain dynamics of low molecular weight polymer melts can be described in terms of the Rouse model. In ad- 
dition to the friction with the surrounding viscous medium and the stochastic forces acting on the segment of polymer chain, this model assumes intramolecular entropic forces between the neighboring segments resulting from the chain conformational entropy. ${ }^{13}$

The Rouse formalism is based on the solution of the Langevin equation for the segments of a Gaussian chain consisting of $N$ segments of length $l$. The solution provides the relaxation spectra of the chain and the dynamical correlation functions. In particular, the mean squared displacement of the segment relative to its initial point can be expressed by following way:

$$
\left\langle r^{2}(t)\right\rangle_{\text {Rouse }}=\frac{2}{\sqrt{\pi^{3}}} l^{2} \sqrt{\frac{t}{\tau_{s}}},
$$

in the limit $\tau_{s} \ll t \ll \tau_{R}$, where $\tau_{s}=l^{2} \zeta_{0} / 3 \pi^{2} k_{B} T$ is the segmental relaxation time, $\tau_{R}=N^{2} \tau_{s}$ is the longest relaxation time (the Rouse time), and $\zeta_{0}$ is the monomeric friction coefficient.

Considering Gaussian distributions for the atomic displacements, the incoherent scattering function is described by the relation

$$
S_{\text {inc }}(Q, t)=\exp \left(-\frac{Q^{2}}{6}\left\langle r^{2}(t)\right\rangle\right) .
$$

Substituting the result of the Rouse model [Eq. (5)] in Eq. (6), $S_{\text {inc }}(Q, t)$ takes the form of a KWW function [Eq. (3)] with $\beta=0.5$ and a relaxation time defined by the formula

$$
\tau_{\mathrm{KWW}}^{\mathrm{Rouse}}(Q, T)=\frac{9 \pi}{W l^{4}} Q^{-4},
$$

where $W=\left(\tau_{s} \pi^{2}\right)^{-1}$ is elementary Rouse frequency characterizing the relaxation rate of the segment. So, extracting the value of $W l^{4}$ from the $Q$-dependent characteristic timescales [Eq. (7)], the temperature dependent monomeric friction coefficient can be derived,

$$
\zeta_{0}=\frac{3 k_{B} T l^{2}}{W l^{4}}
$$

We note that the Rouse model should be applicable only at sufficiently low $Q$-values (where the microscopic potentials cease to be important). In practice, the crossover between Rouse dynamics and local segmental dynamics is difficult to identify and the understanding of the polymer dynamics at these (intermediate) length scales is still an open question. $^{17,20,21}$

\section{Reptation theory}

Dynamics of polymer melts with molecular weight larger than a critical value essentially differs from Rouse dynamics. In such case the uncrossibility effects, or entanglements, come into play. Reptation theory postulates that the displacement of polymer chain segments is effectively confined by a tube formed by the mutually interpenetrable chains. Following the works of de Gennes and Doi and Edwards, ${ }^{8}$ four dynamical regimes can be distinguished. Regime I for short times $\left(\tau_{s}<t<\tau_{e}\right)$ characterizes the unen-

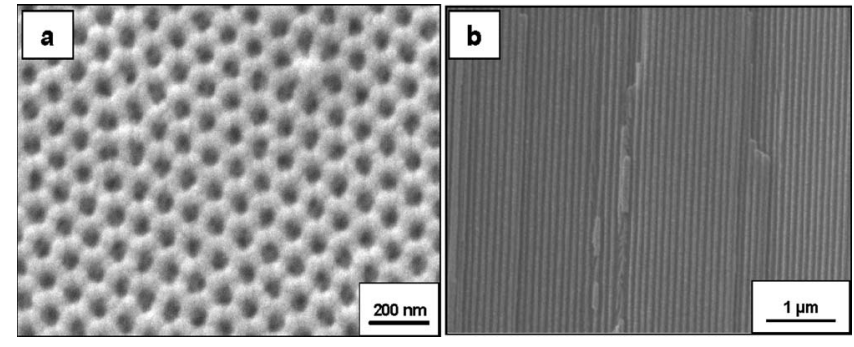

FIG. 1. SEM micrographs of the surface (a) and cross section (b) of the AAO templates.

tangled Rouse dynamics where the entanglements do not yet influence the polymer chain dynamics [Eq. (5)]. The crossover time between unrestricted Rouse and constrained dynamics $\tau_{e}$ (entanglement time) is related to the tube diameter $d$ :

$$
\tau_{e}=\frac{1}{\pi^{2}} \frac{d^{4}}{W l^{4}}
$$

In regime II $\left(\tau_{e}<t<\tau_{R}\right)$ curvilinear Rouse motion of the polymer segments along the tube (local reptation) has to be considered,

$$
\left\langle r^{2}(t)\right\rangle=2 d l\left(\frac{1}{3 \pi^{3}} \frac{t}{\tau_{s}}\right)^{1 / 4} .
$$

Diffusion creep (pure reptation) along the tube, which eventually leads to complete tube renewal, occurs in the regime III $\left(\tau_{R}<t<\tau_{d}\right)$. The mean-square displacement becomes weakly dependent on the molecular weight:

$$
\left\langle r^{2}(t)\right\rangle=2 d l\left(\frac{1}{3 \pi^{2} N} \frac{t}{\tau_{s}}\right)^{1 / 2} .
$$

Regime III is limited by the maximal relaxation time of polymer chain- "disengagement" time $\tau_{d}$. It strongly depends on temperature and the molecular weight of the polymer as $\tau_{d}$ $\sim N^{3}$, as it has been calculated by Doi and Edwards under the assumption of fixed tube. The inclusion of constraint release and contour length fluctuations lead to somewhat higher exponents $(\sim 3.4){ }^{22}$ For long chains $\tau_{d}$ may easily reach the seconds scale. Above $\tau_{d}$ Fickian diffusion of polymer chains is expected with a strong molecular weight dependence of the center of mass displacement $\left\langle r^{2}(t)\right\rangle \sim t N^{-2}$.

\section{EXPERIMENTAL}

\section{A. Samples}

\section{Anodic aluminum oxide templates}

The ordered AAO templates with $40 \mathrm{~nm}$ pore diameter and $150 \mu \mathrm{m}$ pore depth were prepared by a two-step anodization process of aluminum as described elsewhere. ${ }^{23}$ The templates are comprised of a hexagonal array of parallel cylindrical pores oriented perpendicularly to the surface of the template. The morphology of the AAO was characterized by a scanning electron microscope (SEM) (Philips XL-30 ESEM) and is shown in Fig. 1. 


\section{Infiltration of poly(ethylene oxide) into the nanopores}

Films of commercially available and monodisperse PEO (Polymer Source, Inc.; $M_{\mathrm{w}}=43000 \mathrm{~g} \mathrm{~mol}^{-1} ; M_{\mathrm{w}} / M_{\mathrm{n}}=1.05$ ) were prepared from $5 \mathrm{wt} \%$ solution of PEO in chloroform. The average end-to-end distance $R_{\mathrm{e}}$ of PEO polymer chains with $M_{\mathrm{w}}=43000 \mathrm{~g} \mathrm{~mol}^{-1}$ can be calculated to be $19 \mathrm{~nm}^{24}$ The melting temperature of bulk PEO is $338 \mathrm{~K}$. The PEO films were placed onto the AAO templates in order to carry out the infiltration by the "precursor film" infiltration method. ${ }^{25,26}$ In this method, a polymeric liquid precursor film spreads over the whole surface of the AAO template, pore walls included. Since the thickness of the precursor film is larger than the pore radius, a complete filling of the nanopores is achieved. The AAO templates with the PEO film were then annealed at $110{ }^{\circ} \mathrm{C}$ under $\mathrm{N}_{2}$ atmosphere for $60 \mathrm{~min}$. The residual PEO located outside of the pores was mechanically removed using a blade. In order to avoid the absorption of environmental water by the confined PEO and keep the polymer inside the pores, the templates were sealed by aluminum sputtering. Aluminum was chosen as sputtering material because of its transparency to neutrons.

\section{B. Neutron scattering measurements}

For the neutron scattering measurements, six filled templates were placed in a flat aluminum container. Two different orientations of the samples with respect to the incident beam were investigated in all cases $\left(45^{\circ}\right.$ and $135^{\circ}$, see insert in Fig. 6).

\section{DNS}

If the incoherent scattering arises solely from spin disorder, it flips the neutron spin with a probability of $2 / 3$, while coherent scattering leaves the spin unchanged. This allows to experimentally separate the coherent and incoherent contributions to the scattered intensity by using a spin polarized neutron beam and polarization analysis. ${ }^{27}$ For this purpose the diffuse scattering spectrometer DNS of Jülich Centre of Neutron Research (JCNS) at the FRM II has been used. The incident wavelength was set to $4.74 \AA$ enabling a range of the scattering vectors $Q$ between 0.5 and $2.2 \AA^{-1}$. The experiments were performed at room temperature. We measured the intensities scattered by the PEO-filled AAO templates as well as by the empty templates.

\section{Time-of-flight}

The dynamics in the picosecond region was investigated by the ToF spectrometer TOFTOF (Ref. 28) (FRM II) with $\lambda=6.27 \AA$ for the incident beam. We explored five different temperatures from 325 to $400 \mathrm{~K}$. For all of them except for $350 \mathrm{~K}$, where measurements at $135^{\circ}$ are missing, the two orientations were considered. The intensity scattered by the sample at low temperature $(40 \mathrm{~K})$, when all relevant dynamical processes are frozen, has been used as resolution. Background has been taken into account by subtracting the empty aluminum container and the empty AAO templates measurements. Measuring times of about $2.5 \mathrm{~h}$ were used at each $T$ and orientation.
The ToF raw data treatment was carried out with the program IDA. ${ }^{29}$ The data recorded by the detectors in the angular range close to the sample orientation angle were rejected before data reduction because this region was heavily shadowed by the sample. This affected the range $42^{\circ}<2 \vartheta$ $<58^{\circ}$ for nominal $45^{\circ}$ orientation (the angle was actually closer to $48.8^{\circ}$ ) and $2 \vartheta>132^{\circ}$ for nominal $135^{\circ}$ orientation (the angle was actually closer to $135.2^{\circ}$ ). The raw data were normalized to monitor and corrected for detector efficiency. The intensity scattered by the empty templates in the container was subtracted from the total intensity taking into account the transmission of the sample (0.90). Finally, the data were interpolated to constant $Q$ in order to obtain the scattering law $S(Q, \omega)$. Self-shielding corrections were not necessary since for both sample orientations the integrated total intensities turned out to be identical (e.g., $335 \mathrm{~K}$, intensity ratio of reflection and transmission geometry, 1.0014)

\section{Backscattering}

We used the BS instrument SPHERES of the JCNS at the FRM II. The energy window was set to $-31 \mu \mathrm{eV} \leq \hbar \omega$ $\leq 31 \mu \mathrm{eV}$. With $\lambda=6.271 \AA$ and 15 detectors at different angles, $Q$-values in the range between 0.17 and $1.84 \AA^{-1}$ can be accessed. The energy resolution of the instrument was $0.65 \mu \mathrm{eV}$ (full width at half maximum). We investigated 325,335 , and $350 \mathrm{~K}$. The intensities scattered by the empty AAO templates in the container were carefully determined for good background correction. The sample at low temperature (close to $4 \mathrm{~K}$ ) was used to determine the resolution. A measuring time of about $22 \mathrm{~h}$ was employed for each temperature and orientation.

In both TOFTOF and SPHERES experiments, data were collected in $1 \mathrm{~h}$ runs in order to check that the sample did not crystallize during the measurements. Indistinguishable results within the uncertainties were obtained for the different individual spectra for a given temperature and orientation of the sample.

Previous experiments on PEO in bulk performed at different instruments were taken as reference for comparison. They include results from the ToF spectrometer FOCUS (Paul Scherrer Institute, Switzerland) (Refs. 30 and 31) and BS spectrometers PI (former BSS spectrometer at the Forschungsreaktor in Jülich) ${ }^{32}$ and IN16 (ILL, Grenoble). ${ }^{33}$ Experimental data for bulk PEO at temperatures lower than $338 \mathrm{~K}$ were not available because of PEO crystallization.

\section{RESULTS}

\section{A. DNS}

Figure 2(a) shows the ratio between coherent and incoherent contributions of the intensity scattered by the AAO templates (without polymer). No Bragg peaks can be identified in the accessed $Q$-range; the short-range order in the templates is reflected by a broad feature that reveals the amorphous nature of the material. The ratio between coherent and incoherent contributions is in the range of 0.2-2.4, indicating that the incoherent cross section is rather close to the coherent one. Since the incoherent scattering from protons is more than an order of magnitude bigger than all other 


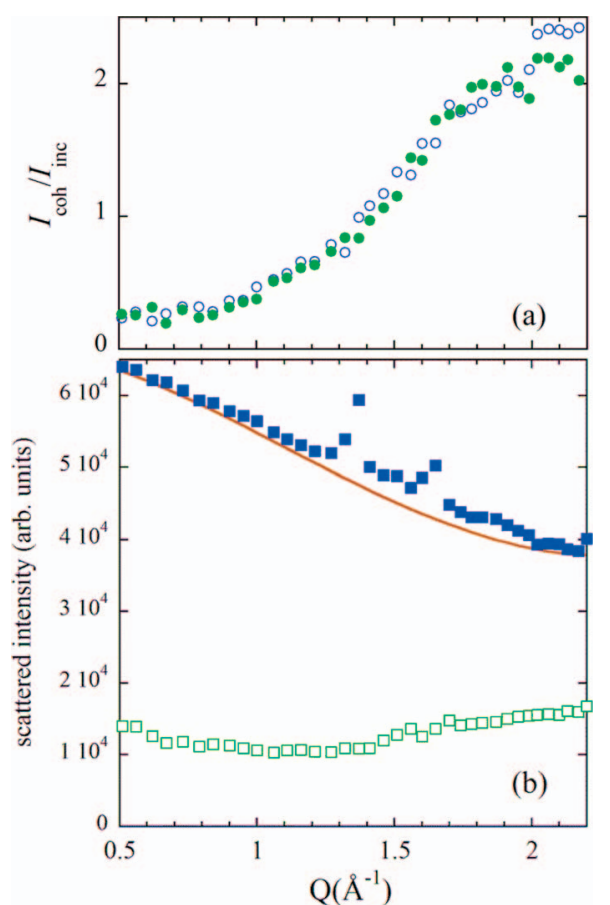

FIG. 2. (a) Ratio between coherent and incoherent contributions to the intensity scattered by the AAO templates; empty circles correspond to $45^{\circ}$ and full circles to $135^{\circ}$ orientation (see insert in Fig. 6). (b) Intensity scattered by PEO in the pores (full symbols) and by the templates and empty cell (empty symbols). The incoherent contribution from PEO hydrogens is shown by the line. The sample was oriented at $135^{\circ}$ with respect to the incident beam. All results are for room temperature.

cross sections, this is only possible if the hydrogen content is very small, and, accordingly, no significant residual water is present in the templates. Figure 2(a) also shows that the system is isotropic at the observed length scales because no dependence on the sample orientation was detected.

The diffraction data also allowed determining the contributions of polymer and container to the total scattered intensity. The symbols in Fig. 2(b) represent the differential cross sections (coherent plus incoherent contributions) measured for both subsystems. The data have been corrected for detector efficiency taking as reference the intensity incoherently scattered by the total system. This intensity was fitted to a function of the form $I_{0} \exp \left[-A Q^{2}+B Q^{4}\right]$ accounting for inelasticity effects. From such description, the factors for correcting the detector efficiency have been calculated and applied to the rest of the results. In the low- $Q$ range, the differential cross section of the sample is five times higher than that of the cell and empty templates. Given the porosity of $9 \%$, the $\mathrm{H}$-density in the matrix may be estimated to be $2 \%$ of that of the polymer. With increasing $Q$, a decrease in the intensity due to inelasticity effects is observed for the polymer. Nevertheless, the line shows that the overwhelming contribution in the whole $Q$-range is the incoherent contribution of the polymer. Finally, the observation of Bragg peaks evidences the semicrystalline structure of PEO at room temperature.

\section{B. Time-of-flight}

Figure 3(a) displays spectra obtained from the PEO confined in AAO nanopores at $375 \mathrm{~K}$ and a sample orientation of

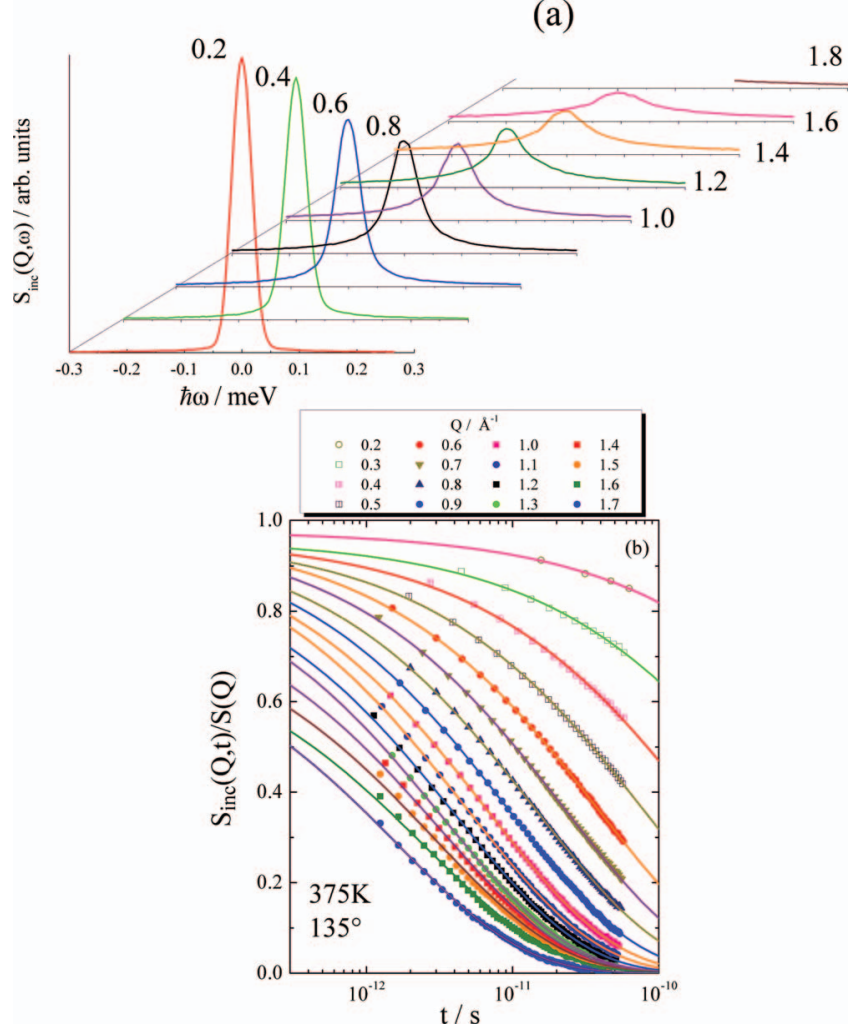

FIG. 3. (a) ToF spectra obtained at $375 \mathrm{~K}$ and $135^{\circ}$ orientation and the different $Q$-values indicated (in $\AA^{-1}$ ). (b) Fourier transformed ToF spectra at the same temperature and orientation. Lines correspond to fits by KWW function [Eq. (3)] with $\beta$ fixed to 0.5 .

$135^{\circ}$ for several $Q$-values in the available region from 0.2 to $1.9 \AA^{-1}$. The energy range where the constant- $Q$ interpolation is possible strongly varies with the $Q$-value. As discussed above, these spectra are dominated by the incoherent contribution reflecting PEO hydrogen motion.

The good statistics and wide energy window accessed by the ToF technique allow Fourier transforming the data into the time domain, where resolution effects can be easily removed dividing by the resolution function and data analysis is more straightforward. Fourier transformed spectra corrected for the resolution function are presented in Fig. 3(b) for $Q$-values from 0.2 to $1.7 \AA^{-1}$. For $Q \geq 1.8 \AA^{-1}$ the spectra were not transformed because they were not defined in the elastic region [see Fig. 3(a)]. Fourier transformed spectra of PEO in AAO for $Q=0.7 \AA^{-1}$ at sample orientation of $45^{\circ}$ and different temperatures are presented in Fig. 4.

\section{Backscattering}

Figure 5 displays typical spectra obtained at SPHERES for different $Q$ values at $350 \mathrm{~K}$. Though the available $Q$-range of SPHERES is $0.17 \AA^{-1}<Q<1.84 \AA^{-1}$, the effective range where useful information can be extracted in our case is rather reduced. On the one hand, the bad resolution of the small-angle detectors limits the range to $Q$ $>0.3 \AA^{-1}$. On the other side, the detected fast dynamics at high $Q$ leads to completely flat spectra above $Q=0.96 \AA^{-1}$ 


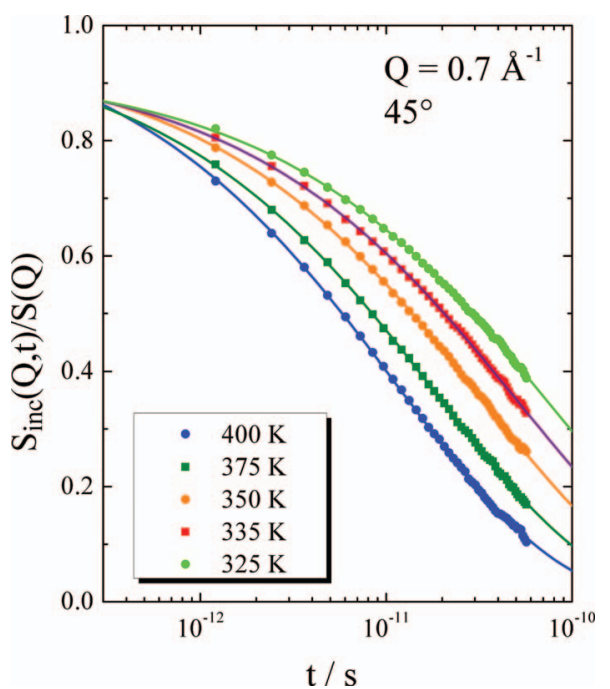

FIG. 4. Fourier transformed ToF spectra obtained at $Q=0.7 \AA^{-1}$ and $45^{\circ}$ orientation of the sample and different temperatures. Lines correspond to fits by KWW function [Eq. (3)] with $\beta$ fixed to 0.5 .

(see Fig. 5). Due to the limited energy window covered by $\mathrm{BS}$ techniques, the data were treated in the frequency domain.

\section{DATA ANALYSIS AND DISCUSSION}

\section{A. Analysis of time-of-flight spectra}

In a first step, each Fourier transformed spectrum [Figs. 3(b) and 4] was fitted by Eq. (3) for $t \geq 2$ ps using three free parameters $\left(\beta, \tau_{\mathrm{KWw}}\right.$, and $\left.A(Q)\right)$. The values of the stretching parameter $\beta$ were found to be close to 0.5 , typical for bulk PEO. In a next step $\beta$ was fixed to 0.5 and the fits were performed with $\tau_{\mathrm{KWw}}$ and $A(Q)$ as free parameters. The fitting curves give an overall good description of the experimental data [see, e.g., Figs. 3(b) and 4].

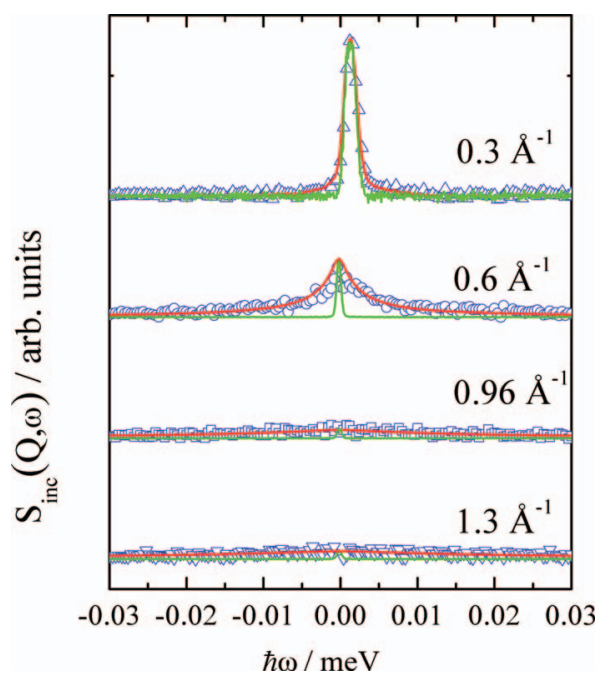

FIG. 5. BS results corresponding to $350 \mathrm{~K}$ at $135^{\circ}$ and different $Q$-values (symbols). The dashed lines show the resolution function. The solid lines are descriptions with the Fourier transformation of Eq. (3) with $\beta=0.5$ and the timescale fixed as obtained from the ToF analysis (see the text).

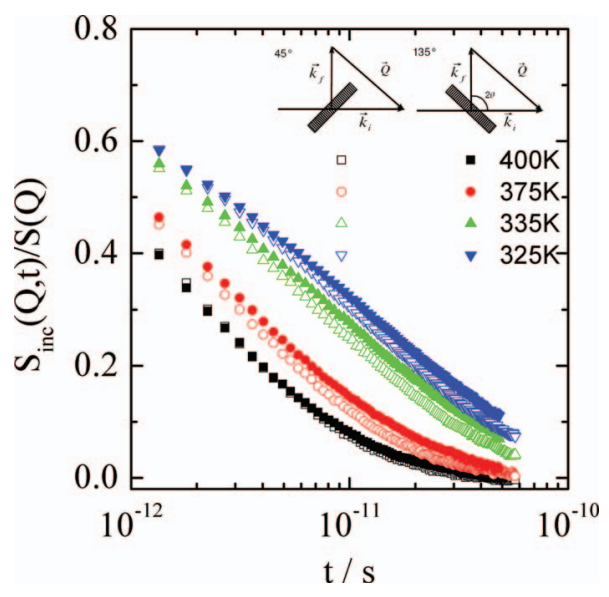

FIG. 6. Comparison between the intermediate scattering functions obtained for the two orientations investigated at different temperatures. The selected $Q$-value $1.4 \AA^{-1}$ corresponding to a scattering angle $2 \vartheta$ of about $90^{\circ}$ relates to $\vec{Q}$ directed along the pores for $45^{\circ}$ orientation of the sample and perpendicular to the pore axis for $135^{\circ}$ orientation (see insert).

\section{B. Analysis of backscattering spectra}

The BS spectra were described in terms of the Fourier transformed stretched exponential function [Eq. (3)] convoluted with the experimental instrumental resolution. The stretching parameter $\beta$ was fixed to 0.5 , and both $\tau_{\mathrm{KWw}}$ and $A(Q)$ were considered as free, as in the case of ToF data analysis. As commented previously, it was not possible to extract reliable information for $Q$-values above approximately $1 \AA^{-1}$. It is worth emphasizing that we find a perfect agreement between the BS and ToF results. This is demonstrated in Fig. 5, where the solid lines have been calculated with the timescales directly deduced from the ToF analysis.

\section{Dependence of the results on the sample orientation}

We first discuss whether the results depend on the orientation of the samples related to the incident beam (insert in Fig. 6). For both instruments, in the $Q$-range below approximately $1 \AA^{-1}$ the spectra recorded at both orientations are indistinguishable, within the experimental uncertainties. The optimum scattering angle to realize about the anisotropy of the motion is $2 \vartheta=90^{\circ}$. There, with the two sample orientations, we explore the components of momentum transfer corresponding to molecular displacements perpendicular (orientation of $135^{\circ}$ ) and parallel (orientation of $45^{\circ}$ ) to the pore axis separately. With the wavelength used, this corresponds to a $Q$-value of $1.4 \AA^{-1}$. For this $Q$-value, the dynamics is too fast to be resolved with SPHERES, but is well observed by TOFTOF. Figure 6 shows the results obtained for the two orientations at the available temperatures. Slight differences in the decays can be observed at $375 \mathrm{~K}$ and below. Taking into account that these experimental conditions are the ideal to resolve an anisotropy in the system, we expect that this difference marks the highest limit for anisotropic effects induced by the confining matrix. 


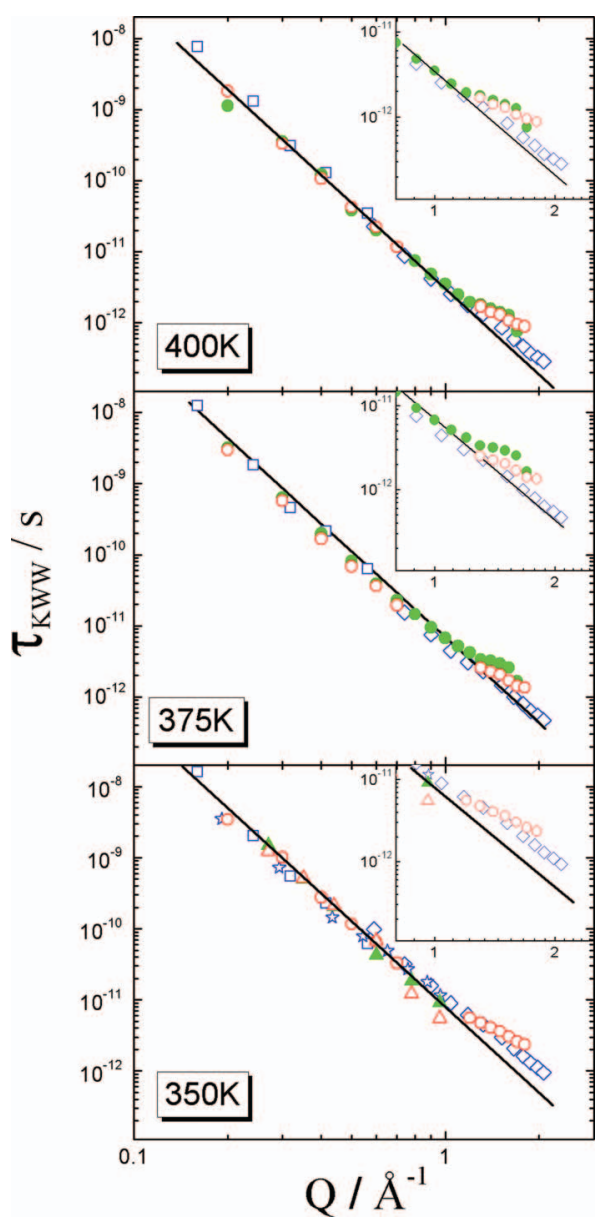

FIG. 7. $Q$-dependence of the characteristic times at 400,375 , and $350 \mathrm{~K}$. Data for bulk PEO are presented by open diamonds (FOCUS), open squares (PI), and open stars (IN16). TOFTOF data at the $45^{\circ}$ and $135^{\circ}$ sample orientations are shown by open and filled cycles, respectively. SPHERES data at the $45^{\circ}$ and $135^{\circ}$ sample orientations are presented by open and filled triangles, respectively. The solid lines show a $Q^{-4}$ dependence (Rouse behavior)

\section{Comparison with bulk PEO}

For a quantitative discussion, we consider the timescales obtained from the KWW description. Figure 7 shows the results obtained for PEO in AAO templates from both ToF and BS instruments. Supporting the consistency of the data analysis performed, the timescales deduced from both spectrometers are in excellent agreement for all temperatures and orientations available. In the same figure the results reported for bulk PEO at different temperatures are also shown for comparison. In the low $Q$ region up to $1 \AA^{-1}$, bulk and confined PEO results are indistinguishable within the uncertainties. In this region, the $Q$-dependence of the relaxation times is well described by the Rouse prediction [Eq. (7)] (see, e.g., Ref. 32). At higher $Q$-values deviations from Rouse behavior for both confined and bulk PEO are obvious, reflecting nonGaussian statistics of the molecular displacements at small lengthscales. This crossover to a weaker $Q$-dispersion has been observed in several polymers and can be rationalized, e.g., in terms of a sublinear jump diffusion model. ${ }^{19,34}$

For the confined polymer we observe that the $Q$-dependence of the characteristic times above $1 \AA^{-1}$ is even weaker than in the bulk. Moreover, the results in the
TABLE I. Characteristic timescales determined by the fitting of the ToF spectra presented in the Fig. $6\left(Q=1.4 \AA^{-1}\right)$ for different temperatures and sample orientations (see insert in Fig. 6).

\begin{tabular}{cccc}
\hline \hline $\begin{array}{c}T \\
(\mathrm{~K})\end{array}$ & $\begin{array}{c}\tau_{\mathrm{KWw}}^{\|} \\
(\mathrm{ps})\end{array}$ & $\begin{array}{c}\tau_{\mathrm{KWW}}^{\perp} \\
(\mathrm{ps})\end{array}$ & $\begin{array}{c}\tau_{\mathrm{KWW}}^{\text {bulk }} \\
(\mathrm{ps})\end{array}$ \\
\hline 325 & 9.56 & 12.2 & $\ldots$ \\
335 & 6.15 & 8.42 & $\ldots$ \\
350 & 4.07 & $\ldots$ & 3.74 \\
375 & 2.26 & 3.20 & 1.85 \\
400 & 1.42 & 1.59 & 1.08 \\
\hline \hline
\end{tabular}

Fig. 7 confirm the anisotropy for the displacements of the PEO segments showed above. For $Q>1 \AA^{-1}$ the relaxation timescales associated with the displacements perpendicular to the pore axis $\tau_{\mathrm{KWw}}^{\perp}$ systematically exceed those attributed to the displacements along the pore axis $\tau_{\mathrm{KWW}}$. In particular, at $Q=1.4 \AA^{-1}$ and $375 \mathrm{~K} \tau_{\mathrm{KWw}}^{\perp}$ exceeds $\tau_{\mathrm{KWw}}$ by a factor of 1.4 and $\tau_{\mathrm{KWW}}^{\mathrm{bulk}}$ by a factor of 1.7 (see Table I). Thus, confinement affects local motions and in a more pronounced way in the direction perpendicular to the pores.

Figure 8 shows the direct comparison between the intermediate scattering function of the confined PEO hydrogens (in perpendicular orientation) and bulk PEO hydrogens at $1.4 \AA^{-1}$ and $375 \mathrm{~K}$. The confinement effect shows up as a further stretching of the decay in the long time regime. Quantification of this effect is not easy; in a first approach we may consider that only a fraction of the hydrogens is affected by confinement. From the integral difference between the two spectra we would deduce a value of $13 \%$ for this fraction. We assume that the confinement effect manifests in a more stretched decay of their correlation function-pronounced stretching is one of the wellestablished features of confined systems. ${ }^{3}$ Assuming a $\beta$-value of 0.25 for this fraction we would deduce that about $13 \%$ of protons would move more slowly (about three times) than bulklike hydrogens (see Fig. 8). Even though the data description is very satisfactory, another functional form for the slow fraction cannot be excluded.

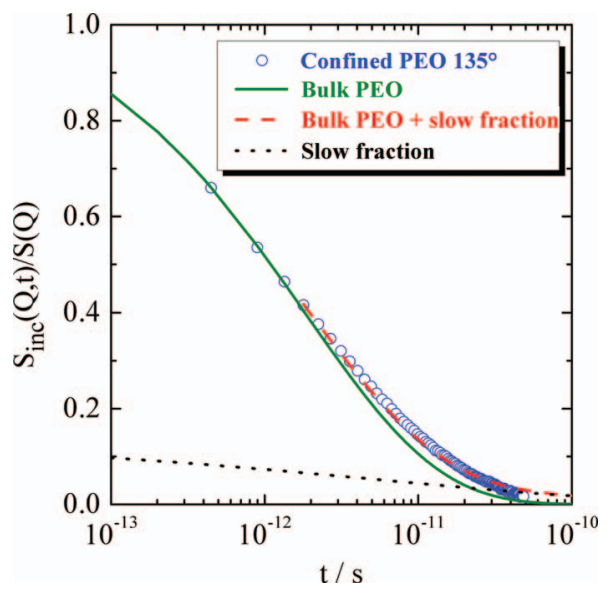

FIG. 8. Intermediate scattering function of PEO hydrogens (circles) in the AAO templates oriented at $135^{\circ}$ and that deduced for bulk PEO (solid line) (Ref. 31) at $375 \mathrm{~K}$ and $Q=1.4 \AA^{-1}$. The dashed line presents a fit by a sum of $87 \%$ of the fast component $\left(\tau_{\mathrm{KWw}}=3.2 \mathrm{ps}\right)$ with $\beta=0.5$ like in a bulk and $13 \%$ slow component $\left(\tau_{\mathrm{KWW}}=9.6 \mathrm{ps}\right)$ with $\beta=0.25$. The slower component is shown by dotted line. 


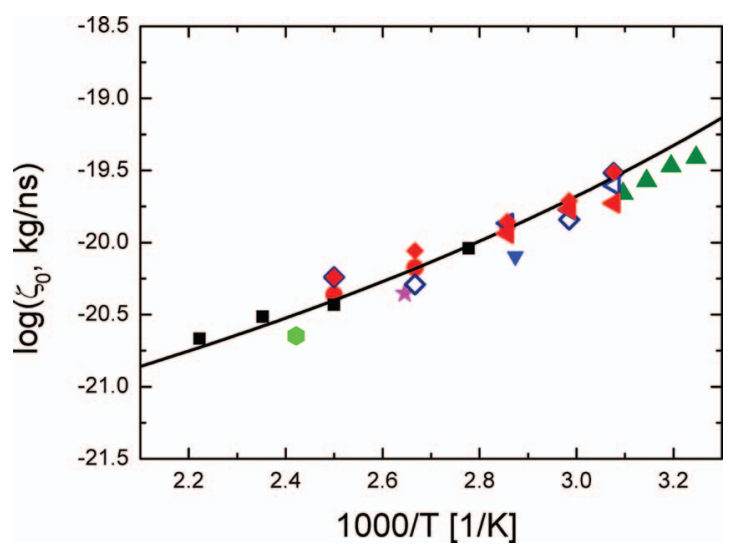

FIG. 9. Temperature dependence of the local friction coefficient of PEO in the pores and in the bulk compared with results of the literature. The symbols correspond to the different molecular weight samples: squares, $M_{\mathrm{w}}$ $=81 \mathrm{~kg} / \mathrm{mol}\left[\mathrm{NSE}\right.$ (Ref. 15)]; circles, $M_{\mathrm{w}}=24 \mathrm{~kg} / \mathrm{mol}$ [backscattering (Ref. 32)]; triangles up, $M_{\mathrm{w}}=0.89 \mathrm{~kg} / \mathrm{mol}$ [rheology (Ref. 15)]; triangle down, $M_{\mathrm{w}}=610 \mathrm{~kg} / \mathrm{mol}\left[\right.$ rheology (Ref. 15)]; star, $M_{\mathrm{w}}=932 \mathrm{~kg} / \mathrm{mol}[\mathrm{rhe}-$ ology (Ref. 15)]; hexagon, $M_{\mathrm{w}}=2.1 \mathrm{~kg} / \mathrm{mol}$ [NSE (Ref. 15)]. TOFTOF results are presented for $45^{\circ}$ and $135^{\circ}$ orientations by open and filled diamonds, respectively. SPHERES data are shown by open and filled left triangles for $45^{\circ}$ and $135^{\circ}$ orientations, respectively. The VTF law [Eq. (12)] is shown by the solid line.

\section{E. Monomeric friction coefficient}

Taking into account $l^{2}=33.75 \AA^{2}$ for PEO (Ref. 35) and using Eq. (7) for data extrapolation in the low $Q$ limit (Fig. 7), the monomeric friction coefficient was calculated from the results of PEO in the pores for the different temperatures investigated [Eq. (8)]. As can be seen in Fig. 9, the resulting values are in good agreement with previous results from different scattering methods as well as rheological data. The temperature dependence of the segmental friction coefficient is well described by Vogel-Tammann-Fulcher (VTF) law:

$$
\zeta_{0}=\zeta_{0}^{\infty} \exp \left(\frac{B}{T-T_{\infty}}\right)
$$

where $B$ is the VTF parameter and $T_{\infty}$ is the VTF temperature. The parameters $T_{\infty}=155 \mathrm{~K}, \zeta_{0}^{\infty}=4.65 \times 10^{-23} \mathrm{~kg} \mathrm{~ns}^{-1}$, and $B=1090 \mathrm{~K}$, taken from the Ref. 15 , have been used. We note that confining PEO melt in AAO templates does not noticeably change the monomeric friction coefficient as compared to bulk PEO.

\section{F. Comparison with NMR and molecular dynamics simulations results}

The neutron scattering experimental techniques used in this work probe the polymer dynamics on timescales from about 1 ps to a few nanoseconds. In fact, at the smallest $Q$-values $\left(0.2-0.4 \AA^{-1}\right.$ for ToF technique presented here) that approximately correspond to distances of order of $\mathrm{Ku}-$ hn's segment, the results can be compared with those obtained by NMR diffusometry and relaxometry. In particular, the experimental data measured with fringe field gradient NMR diffusometry ${ }^{36}$ and proton spin-relaxation data ${ }^{37}$ can be considered for comparison. This might be performed through the mean squared displacements deduced from the different techniques.

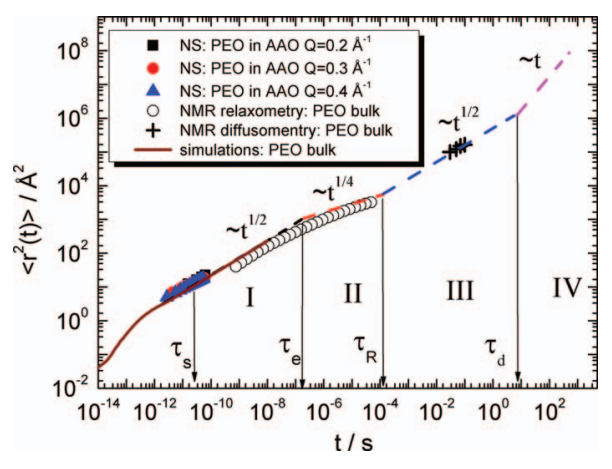

FIG. 10. Mean-squared displacement obtained by neutron scattering (ToF), MD simulations (Ref. 31), fringe field gradient NMR, and field-cycling NMR relaxometry (circles) (Refs. 36 and 37). Neutron scattering data were calculated from ToF spectra of PEO $\left(M_{\mathrm{w}}=43000 \mathrm{~g} \mathrm{~mol}^{-1}\right)$ in AAO pores for $Q=0.2,0.3$, and $0.4 \AA^{-1}$ at $350 \mathrm{~K}$ using the relation $\left\langle r^{2}(t)\right\rangle$ $=-6 \ln (S(Q, t)) / Q^{2}$. Both NMR experiments have been performed for bulk PEO at $355 \mathrm{~K}$ and molecular weight $M_{\mathrm{w}}=178000 \mathrm{~g} \mathrm{~mol}^{-1}$. The vertical arrows indicate dynamical limits of reptation theory that correspond to (I) unrestricted Rouse motion $\sim t^{1 / 2}$, (II) local reptation $\sim t^{1 / 4}$, (III) collective motion of the polymer chains along the tube contour $\sim t^{1 / 2}$, and (IV) normal diffusion of polymer chain for the molecular weight $M_{\mathrm{w}}$ $=178000 \mathrm{~g} \mathrm{~mol}^{-1}$. The characteristic times have been calculated in accordance with reptation/tube model: entanglement time $\tau_{e}=2 \times 10^{-7} \mathrm{~s}$, Rouse relaxation time $\tau_{R}=1.3 \times 10^{-4} \mathrm{~s}$, and terminal relaxation time taking into account constraint release (CR) events (see Sec. II) $\tau_{d}=7 \mathrm{~s}$. MD simulations results of bulk PEO $\left(M_{\mathrm{w}}=1894 \mathrm{~g} \mathrm{~mol}^{-1}\right)$ at $350 \mathrm{~K}$ are presented by a solid line.

In the case of neutron scattering we have seen that for small $Q$-values confined PEO follows Rouse-like dynamics, implying that the Gaussian approximation for the molecular displacements is well fulfilled. In this case without any approximation the intermediate scattering function directly measures the mean-squared displacement [Eq. (6)].

Figure 10 compares our ToF results with those derived from NMR relaxometry ${ }^{37}$ and diffusometry ${ }^{36}$ for bulk PEO. Taking into account the difference in $5 \mathrm{~K}$ in the temperature scale, the data are in good agreement. In order to compare with the corresponding NMR experiments performed at a molecular weight of $178000 \mathrm{~g} \mathrm{~mol}^{-1}$, we calculated DoiEdwards predictions [Eqs. (5), (10), and (11)] for that molecular weight. The results are shown in Fig. 10 as dashed lines. The ToF data are in good agreement with the Rouse prediction [Eq. (5)] in the available experimental range. The NMR relaxometry data cover the crossover region from regime I of Rouse dynamics to regime II of local reptation at $\tau_{e} \ll t \ll \tau_{R}$. The Rouse time $\tau_{R}$ is estimated to be $0.13 \mathrm{~ms}$ for PEO with the given molecular weight. The NMR fieldgradient diffusometry data agree with the reptation regime III. The terminal time $\tau_{d}$ has been estimated to be about $7 \mathrm{~s}^{21}$

The ToF data cover the time interval in the vicinity of the segmental relaxation time $t \sim \tau_{s}$ where the Rouse theory is at its limits. We can compare our data with recent computer simulation results which also cover this range ${ }^{31}$ (see Figs. 10 and 11). In Fig. 11 the mean-squared displacements obtained by the simulation are presented together with the ToF data for different temperatures. At $400 \mathrm{~K}$, the segmental relaxation time $\tau_{s}$ is minimal providing the broadest region for the comparison between experimental data and Rouse theory. The ToF data and simulation curves are in excellent agreement. We thus may expect that the bulk results obtained 


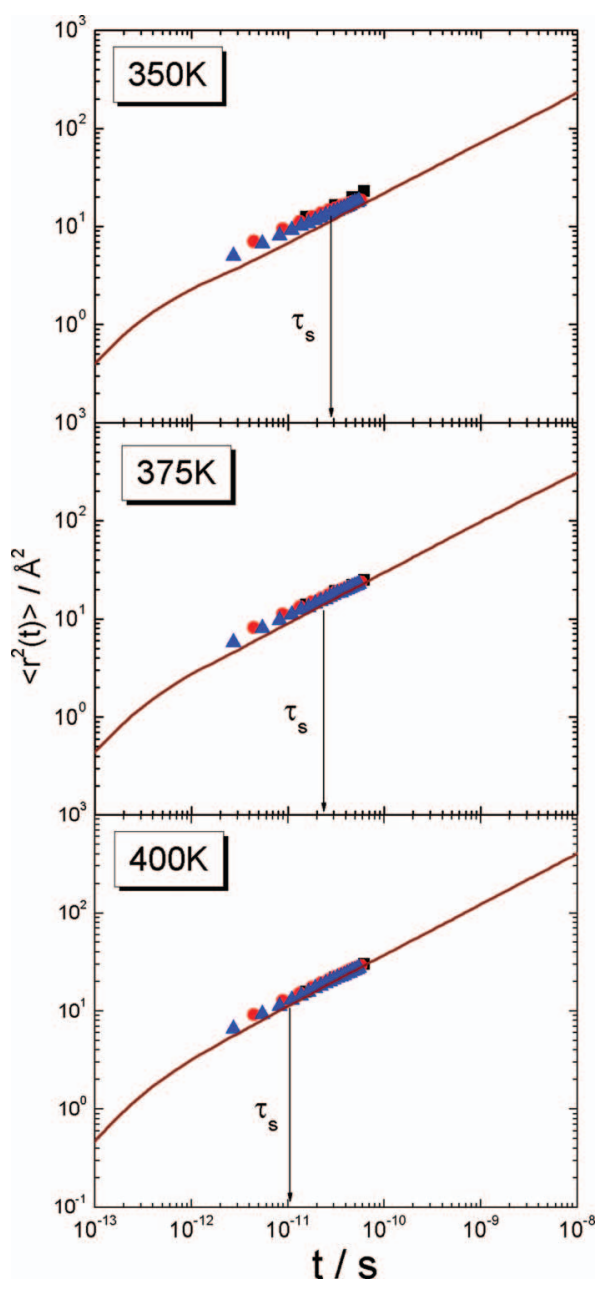

FIG. 11. Mean-squared displacement obtained from neutron scattering (ToF) and MD simulations (Ref. 31) at 350, 375, and $400 \mathrm{~K}$. MD simulations of bulk PEO $\left(M_{\mathrm{w}}=1894 \mathrm{~g} \mathrm{~mol}^{-1}\right)$ are presented by solid lines. ToF results for PEO of molecular weight $M_{\mathrm{w}}=43000 \mathrm{~g} \mathrm{~mol}^{-1}$ in AAO pores for $Q=0.2,0.3$, and $0.4 \AA^{-1}$ are shown by squares, circles, and triangles, respectively. Segmental relaxation time $\tau_{s}$ is $0.028 \mathrm{~ns}$ at $350 \mathrm{~K}, 0.022 \mathrm{~ns}$ at $375 \mathrm{~K}$, and $0.011 \mathrm{~ns}$ at $400 \mathrm{~K}$.

from those simulations ${ }^{31}$ in the overlapping region are extensive to PEO confined in the $40 \mathrm{~nm}$ pores. In that work it was shown that the incoherent scattering function followed well Rouse-like dynamics down to very fast times, in accordance with neutron scattering experiments in the bulk. A detailed analysis of the simulated single chain structure factor in terms of Rouse correlators revealed however deviations from Rouse theory. With respect to the Gaussian behavior of the displacements, the simulations deliver very small values for the second order non-Gaussian parameter, indicating that the Gaussian approximation is well fulfilled in the time regime above some picoseconds.

NMR results for the dynamics of polymer chains confined in different nanoporous structures were interpreted as showing striking differences from bulk dynamics. The earliest works were performed with PEO confined in polyhydroxyethylmethacrylate (PHEMA) solid matrix ${ }^{38,39}$ of 10-15 nm pore diameter. The later experiments considered a larger variety of pore diameters of the PHEMA matrix ${ }^{40}$ from 9 to $57 \mathrm{~nm}$. In all cases the effect of confinement was noticeable on the time-/lengthscale' of the NMR experiment.
Theoretically based on thermodynamic arguments, the characteristic lengthscale of the confinement effect was found to be $0.5 \mathrm{~nm} .{ }^{14}$ On the other side, the crossover from confined to bulk behavior was observed at lengthscales a few orders of magnitude larger than $0.5 \mathrm{~nm}$. From those results it was concluded that impenetrable pore walls, low compressibility of polymer melt, and uncrossibility of polymer chains induce strong restrictions for the polymer chain dynamics. These experimental findings were explained by the corset effect which should take place at the length scale of neutron scattering experiment. In the following we will make an attempt to analyze our experimental data in terms of the corset effect.

\section{G. Corset effect}

As soon as the segment displacement is confined to a curvilinear motion, the Gaussian form of the intermediate scattering function [Eq. (6)] is violated. Considering the Gaussian displacement probability density of the segment along the tube, Fatkullin calculated the proper incoherent structure factor in the three-dimensional Euclidean space arriving at

$$
S^{\text {self }}(Q, t)=\exp \left(\frac{Q^{4} d^{2}}{72}\left\langle s^{2}(t)\right\rangle\right) \operatorname{erfc}\left(\frac{Q^{2} d}{6 \sqrt{2}} \sqrt{\left\langle s^{2}(t)\right\rangle}\right),
$$

where

$$
\left\langle s^{2}(t)\right\rangle=\frac{2 \frac{D_{0}}{N} t}{1+\frac{12 D_{0} t}{N L^{2}}}+\frac{2 l \sqrt{D_{0} t}}{\sqrt{3 \pi}+18 \frac{\sqrt{D_{0} t}}{N l}}
$$

is the mean-squared displacement measured in curvilinear coordinates along the tube axis, $d$ is the tube diameter, $D_{0}$ $=k_{B} T / \zeta_{0}$ is the segmental diffusion coefficient and $L$ $=N l^{2} / d$ is the tube length. ${ }^{41}$ In the limit $t \ll N^{2} l^{2} / D_{0}$ the curvilinear mean-squared displacement [Eq. (14)] is reduced to the Rouse approximation [Eq. (5)] and gives the incoherent scattering function described by Eq. (6). Assuming $d$ as the characteristic lengthscale of the corset effect (tube diameter), we can calculate the incoherent structure factor for the confined system using Eq. (13). One should note that Eqs. (13) and (14) are strictly valid only in the limit $\tau_{e}<t<\tau_{d}$, i.e., when the segmental displacement exceeds the tube diameter. Figure 12 presents the prediction of Eq. (13) for various values of tube diameter $d$ together with the experimental data for $Q=0.6 \AA^{-1}$ at $375 \mathrm{~K}$ for both confined and bulk PEO. The predicted slowing down of the dynamics strongly depends on $d$. Obviously, the experimental results presented here are not compatible with corset effects of associated characteristic length scales of either 0.5 or $1 \mathrm{~nm}$. On the other side, the spectral shape of the experimental data perfectly coincides with the Rouse prediction. Taking into account that the entanglement time $\tau_{e} \propto d^{4}$ strongly depends on the tube diameter, the observation of corset effect in the ToF window would imply smaller tube diameters $(0.5 \mathrm{~nm}$ and less in our case). Comparing the neutron scattering results with the scattering function for the segmental movement in a tube of $0.5-1 \mathrm{~nm}$ and the Rouse scattering function, we may 


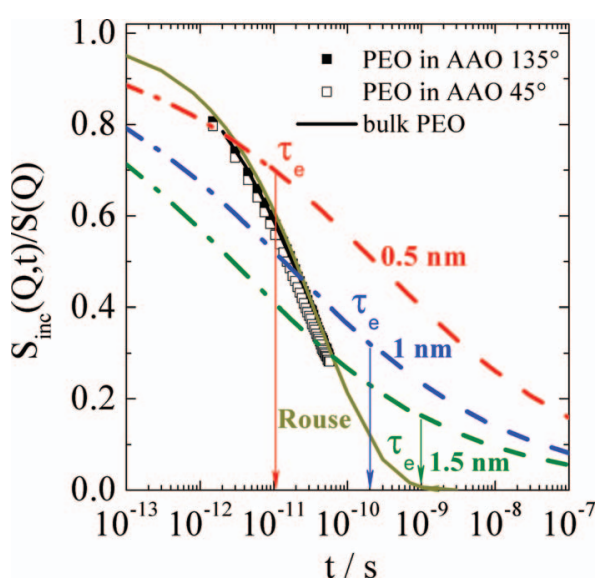

FIG. 12. Comparison of ToF data for PEO in AAO pores (both sample orientations) and bulk PEO $Q=0.6 \AA^{-1}$ at $375 \mathrm{~K}$ with reptation model predictions [Eq. (13)] calculated for tube diameter of $0.5,1$, and $1.5 \mathrm{~nm}$ relating to entanglement time $\tau_{e}$ of $0.012,0.19$, and $0.98 \mathrm{~ns}$, respectively. The function is represented by a dashed line above $\tau_{e}$ (i.e., in the applicability region) and by a dash-dotted line below $\tau_{e}$. Rouse self-correlation function is presented by the solid line.

exclude a corset effect on this time-/lengthscale. Only tube diameters above $\sim 1.5 \mathrm{~nm}$ could be compatible with our experimental results (see Fig. 12).

\section{H. Origin of the confinement effects observed}

The observed slight slowing down of polymer dynamics in the AAO nanopores could be caused by the following phenomena: (i) interactions between the pore walls and polymer segments and (ii) a decrease in the number of conformational transitions within the pore space. The latter is connected to the ability of the system to fluctuate with respect to the free volume needed for conformational rearrangements by the chain modes.

On the time-/lengthscale of the BS experiment within the experimental error the dynamics is well described by Rouse model. Neither an influence of surface interactions of the polymer segments with the pore walls nor any indication of the corset effect was observed. At higher $Q$, the ToF data show some indications for a reduced mobility. This effect is more important for the molecular displacements perpendicular to the pore axis. A detailed analysis of the spectra for $Q$ above $1 \AA^{-1}$ reveals an extra slow component with respect to the bulk. We have seen in Fig. 8 that for $Q=1.4 \AA^{-1}$ and molecular displacements perpendicular to the pore axis, the data can be described by considering a fraction of $13 \%$ of polymer segments with slower response. Attributing this slow fraction to the amount of polymer close to the surface, this value corresponds to a polymer layer of $0.7 \mathrm{~nm}$ thickness. Such surface layer thickness of PEO comprises one to two molecular monolayers. The consideration of similar experiments with PEO under confinement supports this scenario. For PEO confined in Vycor porous glass of $7 \mathrm{~nm}$ pore diameter, the amount of polymer close to the wall is of the order of $50 \% .^{42}$ In that case, a pronounced slowing down of the dynamics at the time-/lengthscale of ToF experiment has been reported. The effect of the confinement on the polymer dynamics has also been observed by NMR in porous Vycor glasses ${ }^{43}$ and @ Anopore membranes. ${ }^{44}$ The last experiments performed with polymer films of poly(dimethyl siloxane) of different thicknesses revealed a most pronounced effect of the confinement for a polymer film of the order of $1 \mathrm{~nm}$.

Corset effect with $d$ of the order of $0.5 \mathrm{~nm}$ as proposed in Ref. 14 can be excluded from our experiments. As one can see from Fig. 11, $0.5 \mathrm{~nm}$ is the maximum average atomic displacement (square root of the mean-squared displacement) that can be deduced by ToF for this system in the temperature range explored. Furthermore, the BS data extend this observation up to $1-2 \mathrm{~nm}$. As we have shown by direct comparison of the scattering function measured and expected from corset effect with different associated tube diameters in the range $0.5-1.5 \mathrm{~nm}$ (see Fig. 12), reductions in the tube diameter down to below $1-1.5 \mathrm{~nm}$ can be excluded. However, the experimental window explored by ToF and BS does not allow extending the study to larger displacements and the existence of corset effect with larger associated tube diameters $(d>1.5 \mathrm{~nm})$ can at present not be discarded. The study of confined polymer chain dynamics on longer time- and lengthscales would certainly add valuable information on confinement effects on the overall chain relaxation. This is a target of forthcoming research by the neutron spin-echo technique that allows extending the timescale of observation up to hundreds of nanoseconds and characteristic lengthscales up to tens of nanometers.

\section{CONCLUSIONS}

The local dynamics of PEO confined in nanopores of AAO has been studied using different neutron scattering techniques. It has been shown by DNS experiments that the AAO has amorphous structure at RT. The polymer chains were found to have semicrystalline structure. No indication of the presence of residual water in the AAO templates was revealed.

The dynamics of the confined polymer is indistinguishable from that in the bulk in the $Q$-range of approximately $0.2 \AA^{-1}<Q<1 \AA^{-1}$. The incoherent scattering function is well described by the Rouse theory showing no indication of confined segmental dynamics due to the corset effect. Accordingly, the monomeric friction coefficient calculated on the basis of the Rouse theory agrees with previous bulk results.

Around $Q=1.4 \AA^{-1}$ (where we probed motions perpendicular and parallel to the pore axis), an anisotropic slowing down of the dynamics is observed under confinement, this effect being more pronounced in the direction perpendicular to the pore axis. The observed slowing down of the molecular displacements perpendicular to the pore axis affects about $\sim 10 \%$ of the segments. This effect could be attributed to the interactions between the pore walls and polymer segments within a $\sim 1 \mathrm{~nm}$ layer.

With the data at hand, we can exclude the existence of a corset effect on the lengthscale where it was proposed from NMR studies. To answer the question whether the additional confinement effects arise at larger lengthscales will need further investigations. In this work we estimated a minimum possible value of the tube diameter of $\sim 1.5 \mathrm{~nm}$ from our 
results in the ToF and $\mathrm{BS}$ window. We expect that future neutron spin-echo experiments addressing the dynamics at larger lengthscales will tell whether there exists a change in chain confinement beyond the tube diameter in the pores.

\section{ACKNOWLEDGMENTS}

The European Commission, NMI3 Contract No. RII3CT-2003-505925, supported the experiments. Financial support from NoE SoftComp (Contract No. NMP3-CT-2004502235) is gratefully acknowledged. The authors are grateful to R. Kimmich and N. Fatkullin for experimental NMR data and helpful discussions, A. Arenas for the help in the Alumina templates preparation, O. Sanchez for the aluminum sputtering of the templates, and M. Brodeck for computer simulation data. Support from Donostia International Physics Center (DIPC) is also acknowledged as well as Project Nos. MAT2007-63681, MAT2008-1073, and IT-436-07 (GV), and the Spanish Ministerio de Educacion y Ciencia (Grant Nos. CSD2006-53 and FPU AP2005-1063).

${ }^{1}$ Y. Mai and Z. Yu, Polymer Nanocomposites (CRC, Boca Raton, FL, 2006).

${ }^{2}$ M. Zhang, P. Dobriyal, J.-T. Chen, and T. Russell, Nano Lett. 6, 1075 (2006).

${ }^{3}$ M. Koza, B. Frick, and R. Zorn, Eur. Phys. J. Spec. Top. 3, 141 (2007).

${ }^{4}$ J.-M. Zanotti, L. J. Smith, D. L. Price, and M.-L. Saboungi, Mater. Res. Soc. Symp. Proc. 790, 187 (2004).

${ }^{5}$ T. Dollase, R. Graf, A. Heuer, and H. W. Spiess, Macromolecules 34, 298 (2001).

${ }^{6}$ H. D. Rowland, W. P. King, J. B. Pethica, and G. L. W. Cross, Science 322, 720 (2008).

${ }^{7}$ P. G. de Gennes, Scaling Concepts in Polymer Physics (Cornell University Press, Ithaka, 1979).

${ }^{8}$ M. Doi and S. Edwards, The Theory of Polymer Dynamics (Clarendon, Oxford, 1986).

${ }^{9}$ A. Wischnewski, M. Monkenbusch, L. Willner, D. Richter, A. E. Likhtman, T. C. B. McLeish, and B. Farago, Phys. Rev. Lett. 88, 058301 (2002).

${ }^{10}$ P. Schleger, B. Farago, C. Lartigue, A. Kollmar, and D. Richter, Phys. Rev. Lett. 81, 124 (1998).

${ }^{11}$ M. Zamponi, M. Monkenbusch, L. Willner, A. Wischnewski, B. Farago, and D. Richter, Europhys. Lett. 72, 1039 (2005).

${ }^{12}$ M. Zamponi, M. Monkenbusch, L. Willner, D. Richter, A. E. Likhtman, G. Kali, and B. Farago, Phys. Rev. Lett. 96, 238302 (2006).

${ }^{13}$ P. J. Rouse, J. Chem. Phys. 21, 1272 (1953).

${ }^{14}$ N. Fatkullin, R. Kimmich, E. Fischer, C. Mattea, U. Beginn, and M. Kroutieva, New J. Phys. 6, 46 (2004).

${ }^{15}$ K. Niedzwiedz, A. Wischnewski, W. Pyckhout-Hintzen, J. Allgaier, D.
Richter, and A. Faraone, Macromolecules 41, 4866 (2008).

${ }^{16}$ R. Kausik, C. Mattea, N. Fatkullin, and R. Kimmich, J. Chem. Phys. 124, 114903 (2006).

${ }^{17}$ D. Richter, M. Monkenbusch, A. Arbe, and J. Colmenero, Adv. Polym. Sci. 174, 1 (2005)

${ }^{18}$ J. Colmenero, A. Alegria, A. Arbe, and B. Frick, Phys. Rev. Lett. 69, 478 (1992).

${ }^{19}$ A. Arbe, J. Colmenero, F. Alvarez, M. Monkenbusch, D. Richter, B. Farago, and B. Frick, Phys. Rev. E 67, 051802 (2003).

${ }^{20}$ D. Richter, M. Monkenbusch, J. Allgaier, A. Arbe, J. Colmenero, B. Farago, Y. Cheol Bae, and R. Faust, J. Chem. Phys. 111, 6107 (1999).

${ }^{21}$ D. Richter, M. Monkenbusch, L. Willner, A. Arbe, J. Colmenero, and B. Farago, Europhys. Lett. 66, 239 (2004).

${ }^{22}$ T. C. B. McLeish, Adv. Phys. 51, 1379 (2002).

${ }^{23}$ H. Masuda and K. Fukuda, Science 268, 1466 (1995).

${ }^{24}$ N. Heymans, Macromolecules 33, 4226 (2000).

${ }^{25}$ M. Steinhart, J. H. Wendorff, A. Greiner, R. B. Wherspohn, K. Nielsch, J. Schilling, J. Choi, and U. Gösele, Science 296, 1997 (2002).

${ }^{26}$ J. Martín and C. Mijangos, Langmuir 25, 1181 (2009).

${ }^{27}$ S. S. W. Lovesey, Theory of Neutron Scattering from Condensed Matter (Clarendon, Oxford, 1984).

${ }^{28}$ T. Unruh, J. Neuhaus, and W. Petry, Nucl. Instrum. Methods Phys. Res. A 580, 1414 (2007).

${ }^{29} \mathrm{http}: / /$ sourceforge.net/projects/frida

${ }^{30}$ K. Niedzwiedz, A. Wischnewski, M. Monkenbusch, D. Richter, A.-C. Genix, A. Arbe, J. Colmenero, M. Strauch, and E. Straube, Phys. Rev. Lett. 98, 168301 (2007).

${ }^{31}$ M. Brodeck, F. Alvarez, A. Arbe, F. Juranyi, T. Unruh, O. Holderer, J. Colmenero, and D. Richter, J. Chem. Phys. 130, 094908 (2009).

${ }^{32}$ A.-C. Genix, A. Arbe, F. Alvarez, J. Colmenero, L. Willner, and D. Richter, Phys. Rev. E 72, 031808 (2005).

${ }^{33}$ A. Tyagi, A. Arbe, J. Colmenero, B. Frick, and J. R. Stewart, Macromolecules 39, 3007 (2006).

${ }^{34}$ A. Arbe, J. Colmenero, F. Alvarez, M. Monkenbusch, D. Richter, B. Farago, and B. Frick, Phys. Rev. Lett. 89, 245701 (2002).

${ }^{35}$ G. D. Smith, D. Y. Yoon, R. L. Jaffe, R. H. Colby, R. Krishnamoorti, and L. J. Fetters, Macromolecules 29, 3462 (1996).

${ }^{36}$ E. Fischer, R. Kimmich, and N. Fatkullin, J. Chem. Phys. 106, 9883 (1997).

${ }^{37}$ M. Kehr, N. Fatkullin, and R. Kimmich, J. Chem. Phys. 126, 094903 (2007).

${ }^{38}$ E. Fischer, R. Kimmich, U. Beginn, M. Moller, and N. Fatkullin, Phys. Rev. E 59, 4079 (1999).

${ }^{39}$ R. Kimmich, R.-O. Seitter, U. Beginn, M. Moller, and N. Fatkullin, Chem. Phys. Lett. 307, 147 (1999).

${ }^{40}$ C. Mattea, N. Fatkullin, E. Fischer, U. Beginn, E. Anoardo, M. Kroutieva, and R. Kimmich, Appl. Magn. Reson. 27, 371 (2004).

${ }^{41}$ N. Fatkullin and R. Kimmich, Phys. Rev. E 52, 3273 (1995).

${ }^{42}$ J.-M. Zanotti, L. J. Smith, D. L. Price, and M.-L. Saboungi, Ann. Chim. Sci. Mater. 30, 353 (2004).

${ }^{43}$ S. Stapf and R. Kimmich, Macromolecules 29, 1638 (1996).

${ }^{44}$ S. Ayalur-Karunakaran, B. Blümich, and S. Stapf, Eur. Phys. J. E 26, 43 (2008). 